\title{
ON A NEW SEQUENCE SPACE DEFINED BY ORLICZ FUNCTIONS
}

\author{
VAKEEL A. KHAN
}

\begin{abstract}
The sequence space $B V_{\sigma}$ was introduced and studied by Mursaleen [9]. In this paper we extend $B V_{\sigma}$ to $B V_{\sigma}(M, p, r)$ and study some properties and inclusion relations on this space.
\end{abstract}

\section{Introduction}

Let $l_{\infty}$ and $c$ denote the Banach spaces of bounded and convergent sequences $x=\left(x_{k}\right)_{k=1}^{\infty}$ respectively. Let $\sigma$ be an injection of the set of positive integers $\mathbb{N}$ into itself having no finite orbits and $T$ be the operator defined on $l_{\infty}$ by $T\left(\left(x_{n}\right)_{n=1}^{\infty}\right)=\left(x_{\sigma(n)}\right)_{n=1}^{\infty}$.

A positive linear functional $\phi$, with $\|\phi\|=1$, is called a $\sigma$ - mean or an invariant mean if $\phi(x)=\phi(T x)$ for all $x \in l_{\infty}$.

A sequence $x$ is said to be $\sigma$ - convergent, denoted by $x \in V_{\sigma}$, if $\phi(x)$ takes the same value, called $\sigma-\lim x$, for all $\sigma$ - means $\phi$. We have (see Schaefer [14])

$$
V_{\sigma}=\left\{x=\left(x_{n}\right): \sum_{m=1}^{\infty} t_{m, n}(x)=L \text { uniformly in } \mathrm{n}, L=\sigma-\lim x\right\},
$$

where for $m \geq 0, n>0$

$$
t_{m, n}(x)=\frac{x_{n}+x_{\sigma(n)}+\cdots+x_{\sigma^{m}(n)}}{m+1}, \text { and } t_{-1, n}=0 .
$$

where $\sigma^{m}(n)$ denotes the $\mathrm{m}$ th iterate of $\sigma$ at $n$. In particular, if $\sigma$ is the translation, a $\sigma$ - mean is often called a Banach limit and $V_{\sigma}$ reduces to f , the set of almost

Received by the editors Nov. 01, 2007; Accepted: Sept. 05, 2008.

2000 Mathematics Subject Classification. Primary 40F05, 40C05, 46A45.

Key words and phrases. Invariant mean, Paranorm, Orlicz function, Solid space .

(C)2008 Ankara University 
- convergent sequences (see Lorentz [5]). Subsequently invariant means have been studied by Ahmad and Mursaleen [1] , Mursaleen [8], Raimi [12] and many others.

The concept of paranorm is closely related to linear metric spaces. It is a generalization of that of absolute value. Let $X$ be a linear space. A function $g: X \rightarrow \mathbb{R}$ is called paranorm, if

[P1] $g(x) \geq 0$, for all $x \in X$,

[P2] $g(-x)=g(x)$, for all $x \in X$,

[P3] $g(x+y) \leq g(x)+g(y)$, for all $x, y \in X$,

[P4] If $\left(\lambda_{n}\right)$ is a sequence of scalars with $\lambda_{n} \rightarrow \lambda(n \rightarrow \infty)$ and $\left(x_{n}\right)$ is a sequence of vectors with $g\left(x_{n}-x\right) \rightarrow 0(n \rightarrow \infty)$, then $g\left(\lambda_{n} x_{n}-\lambda x\right) \rightarrow 0(n \rightarrow \infty)$.

A paranorm $g$ for which $g(x)=0$ implies $x=0$ is called a total paranorm on $X$, and the pair $(X, g)$ is called a totally paranormed space. It is well known that the metric of any linear metric space is given by some total paranorm (cf. [15, Theorem 10.4.2, p. 183]).

A map $M: \mathbb{R} \rightarrow[0,+\infty]$ is said to be an Orlicz function if $M$ is even, convex, left continuous on $\mathbb{R}_{+}$, continuous at zero, $M(0)=0$ and $M(u) \rightarrow \infty$ as $u \rightarrow \infty$. If $M$ takes value zero only at zero we will write $M>0$ and if $M$ takes only finite values we will write $M<\infty$. [2,3,6,7,10,13].

W.Orlicz [11] used the idea of orlicz function to construct the space $\left(L^{M}\right)$. Lindendstrauss and Tzafriri [4] used the idea of Orlicz function to define orlicz sequence space

$$
\ell_{M}:=\left\{x \in \omega: \sum_{k=1}^{\infty} M\left(\frac{\left|x_{k}\right|}{\rho}\right)<\infty \text { for some } \rho>0\right\}
$$

in more detail . $\ell_{M}$ is a Banach space with the norm

$$
\|x\|:=\inf \left\{\rho>0: \sum_{k=1}^{\infty} M\left(\frac{\left|x_{k}\right|}{\rho}\right) \leq 1\right\}
$$

The space $l_{M}$ is closely related to the space $l_{p}$, which is an Orlicz sequence space with $M(x)=x^{p}$ for $1 \leq p<\infty$.

The $\triangle_{2}$ - condition is equivalent to

$$
M(L x) \leq K L M(x) \text {, for all values of } x \geq 0 \text {, and for } L>1 .
$$

An Orlicz function $M$ can always be represented in the following integral form

$$
M(x)=\int_{0}^{x} \eta(t) d t,
$$

where $\eta$ is known as the kernel of $M$, is right differentiable for $t \geq 0, \eta(0)=0$, $\eta(t)>0, \eta$ is non-decreasing and $\eta(t) \rightarrow \infty$ as $t \rightarrow \infty$. Note that an Orlicz function 
satisfies the inequality

$$
M(\lambda x) \leq \lambda M(x) \text { for all } \lambda \text { with } 0<\lambda<1 .
$$

Let $E$ be a sequence space . Then $E$ is called

(i) A sequence space $E$ is said to be symmetric if $\left(x_{n}\right) \in E$ implies $\left(x_{\pi}(n)\right) \in E$, where $\pi(n)$ is a permutation of the elements of the elements of $\mathbb{X}$.

(ii) Solid (or normal), if $\left(\alpha_{k} x_{k}\right) \in E$, whenever $\left(x_{k}\right) \in E$ for all sequences of scalars $\left(\alpha_{k}\right)$ with $\left|\alpha_{k}\right| \leq 1$ for all $k \in \mathbb{X}$.

Lemma 1.1. . A sequence space $E$ is solid implies $E$ is monotone.

Mursaleen [9] defined the sequence space

$$
B V_{\sigma}=\left\{x \in l_{\infty}: \sum_{m}\left|\phi_{m, n}(x)\right|<\infty, \text { uniformly in } \mathrm{n}\right\},
$$

where

$$
\phi_{m, n}(x)=t_{m, n}(x)-t_{m-1, n}(x)
$$

assuming that

$$
t_{m, n}(x)=0, \text { for } \mathrm{m}=-1 \text {. }
$$

A straightforward calculation shows that

$$
\phi_{m, n}(x)= \begin{cases}\frac{1}{m(m+1)} \sum_{j=1}^{m} j\left(x_{\sigma^{j}(n)}-x_{\sigma^{j-1}(n)}\right) & (m \geq 1) \\ x_{n}, & (m=0)\end{cases}
$$

Note that for any sequence $x, y$ and scalar $\lambda$ we have

$$
\phi_{m, n}(x+y)=\phi_{m, n}(x)+\phi_{m, n}(y) \text { and } \phi_{m, n}(\lambda x)=\lambda \phi_{m, n}(x) \text {. }
$$




\section{Main Results.}

Let $M$ be an Orlicz function, $p=\left(p_{m}\right)$ be any sequence of strictly positive real numbers and $r \geq 0$. Now we define the sequence space as follows :

$$
B V_{\sigma}(M, p, r)=\left\{\begin{array}{c}
x=\left(x_{k}\right): \sum_{m=1}^{\infty} \frac{1}{m^{r}}\left[M\left(\frac{\left|\phi_{m, n}(x)\right|}{\rho}\right)\right]^{p_{m}}<\infty, \\
\text { uniformly in } \mathrm{n} \text { and for some } \rho>0
\end{array}\right\} .
$$

For $M(x)=x$ we get

$$
B V_{\sigma}(p, r)=\left\{x=\left(x_{k}\right): \sum_{m=1}^{\infty} \frac{1}{m^{r}}\left|\phi_{m, n}(x)\right|^{p_{m}}<\infty, \text { uniformly in } \mathrm{n}\right\} .
$$

For $p_{m}=1$, for all $m$, we get

$$
B V_{\sigma}(M, r)=\left\{\begin{array}{c}
x=\left(x_{k}\right): \sum_{m=1}^{\infty} \frac{1}{m^{r}}\left[M\left(\frac{\left|\phi_{m, n}(x)\right|}{\rho}\right)\right]<\infty, \\
\text { uniformly in } \mathrm{n} \text { and for some } \rho>0
\end{array}\right\} .
$$

For $r=0$ we get

$$
B V_{\sigma}(M, p)=\left\{\begin{array}{c}
x=\left(x_{k}\right): \sum_{m=1}^{\infty}\left[M\left(\frac{\left|\phi_{m, n}(x)\right|}{\rho}\right)\right]^{p_{m}}<\infty, \\
\text { uniformly in } \mathrm{n} \text { and for some } \rho>0
\end{array}\right\} .
$$

For $M(x)=x$ and $r=0$ we get

$$
B V_{\sigma}(p)=\left\{x=\left(x_{k}\right): \sum_{m=1}^{\infty}\left|\phi_{m, n}(x)\right|^{p_{m}}<\infty, \text { uniformly in } \mathrm{n}\right\} .
$$

For $p_{m}=1$, for all $m$ and $r=0$ we get

$$
B V_{\sigma}(M)=\left\{\begin{array}{c}
x=\left(x_{k}\right): \sum_{m=1}^{\infty}\left[M\left(\frac{\left|\phi_{m, n}(x)\right|}{\rho}\right)\right]<\infty, r \geq 0, \\
\text { uniformly in } \mathrm{n} \text { and for some } \rho>0
\end{array}\right\} .
$$

For $M(x)=x, p_{m}=1$, for all $m$, and $r=0$ we get

$$
B V_{\sigma}=\left\{x=\left(x_{k}\right): \sum_{m=1}^{\infty}\left|\phi_{m, n}(x)\right|<\infty, \text { uniformly in } \mathrm{n}\right\} .
$$

Theorem 2.1. The sequence space $B V_{\sigma}(M, p, r)$ is a linear space over the field $\mathbb{C}$ of complex numbers. 
Proof. . Let $x, y \in B V_{\sigma}(M, p, r)$ and $\alpha, \beta \in \mathbb{C}$. Then there exist positive numbers $\rho_{1}$ and $\rho_{2}$ such that

$$
\sum_{m=1}^{\infty} \frac{1}{m^{r}}\left[M\left(\frac{\left|\phi_{m, n}(x)\right|}{\rho_{1}}\right)\right]^{p_{m}}<\infty
$$

and

$$
\sum_{m=1}^{\infty} \frac{1}{m^{r}}\left[M\left(\frac{\left|\phi_{m, n}(y)\right|}{\rho_{2}}\right)\right]^{p_{m}}<\infty, \text { uniformly in } \mathrm{n} .
$$

Define $\rho_{3}=\max \left(2|\alpha| \rho_{1}, 2|\beta| \rho_{2}\right)$. Since $M$ is nondecreasing and convex we have

$$
\begin{gathered}
\sum_{m=1}^{\infty} \frac{1}{m^{r}}\left[M\left(\frac{\left|\alpha \phi_{m, n}(x)+\beta \phi_{m, n}(y)\right|}{\rho_{3}}\right)\right]^{p_{m}} \\
\leq \sum_{m=1}^{\infty} \frac{1}{m^{r}}\left[M\left(\frac{\left|\alpha \phi_{m, n}(x)\right|}{\rho_{3}}+\frac{\left|\beta \phi_{m, n}(y)\right|}{\rho_{3}}\right)\right]^{p_{m}} \\
\leq \sum_{m=1}^{\infty} \frac{1}{m^{r}} \frac{1}{2}\left[M\left(\frac{\phi_{m, n}(x)}{\rho_{1}}\right)+M\left(\frac{\phi_{m, n}(y)}{\rho_{2}}\right)\right]<\infty, \text { uniformly in } \mathrm{n} .
\end{gathered}
$$

This proves that $B V_{\sigma}(M, p, r)$ is a linear space over the field $\mathbb{C}$ of complex numbers.

Theorem 2.2. For any Orlicz function $M$ and a bounded sequence $p=\left(p_{m}\right)$ of strictly positive real numbers, $B V_{\sigma}(M, p, r)$ is a paranormed(need not be total paranormed) space with

$$
g(x)=\inf _{n \geq 1}\left\{\rho^{\frac{p_{n}}{K}}:\left(\sum_{m=1}^{\infty} \frac{1}{m^{r}}\left[M\left(\frac{\left|\phi_{m, n}(x)\right|}{\rho}\right)\right]^{p_{m}}\right)^{\frac{1}{K}} \leq 1, \text { uniformly in } n\right\} .
$$

where $K=\max \left(1, \sup p_{m}\right)$.

Proof. It is clear that $g(x)=g(-x)$. Since $M(0)=0$, we get

$$
\inf \left\{\rho^{\frac{p_{n}}{K}}\right\}=0, \text { for } x=0 .
$$

By using Theorem 1 , for $\alpha=\beta=1$, we get

$$
g(x+y) \leq g(x)+g(y) .
$$

For the continuity of scalar multiplication let $l \neq 0$ be any complex number. Then by the definition we have

$$
g(l x)=\inf _{n \geq 1}\left\{\rho^{\frac{p_{n}}{K}}:\left(\sum_{m=1}^{\infty} \frac{1}{m^{r}}\left[M\left(\frac{\left|\phi_{m, n}(l x)\right|}{\rho}\right)\right]^{p_{m}}\right)^{\frac{1}{K}} \leq 1, \text { uniformly in } \mathrm{n}\right\}
$$




$$
g(l x)=\inf _{n \geq 1}\left\{\begin{array}{c}
(|l| s)^{\frac{p_{n}}{K}}:\left(\sum_{m=1}^{\infty} \frac{1}{m^{r}}\left[M\left(\frac{\left|\phi_{m, n}(l x)\right|}{s|l|}\right)\right]^{p_{m}}\right)^{\frac{1}{K}} \leq 1 \\
\text { uniformly in } \mathrm{n}
\end{array}\right\}
$$

where $s=\frac{\rho}{|l|}$. Since $|l|^{p_{n}} \leq \max \left(1,|l|^{H}\right)$, we have

$$
\begin{gathered}
g(l x) \leq \max \left(1,|l|^{H}\right) \inf _{n \geq 1}\left\{\begin{array}{c}
s^{\frac{p_{n}}{K}}:\left(\sum_{m=1}^{\infty} \frac{1}{m^{r}}\left[M\left(\frac{\left|\phi_{m, n}(x)\right|}{s}\right)\right]^{p_{m}}\right)^{\frac{1}{K}} \leq 1, \\
\text { uniformly in } \mathrm{n}
\end{array}\right\} \\
=\max \left(1,|l|^{H}\right) g(x)
\end{gathered}
$$

and therefore $g(l x)$ converges to zero when $g(x)$ converges to zero in $B V_{\sigma}(M, p, r)$.

Now let $x$ be fixed element in $B V_{\sigma}(M, p, r)$. There exists $\rho>0$ such that

$$
g(x)=\inf _{n \geq 1}\left\{\rho^{\frac{p_{n}}{K}}:\left(\sum_{m=1}^{\infty} \frac{1}{m^{r}}\left[M\left(\frac{\left|\phi_{m, n}(x)\right|}{\rho}\right)\right]^{p_{m}}\right)^{\frac{1}{K}} \leq 1 \text {, uniformly in } \mathrm{n}\right\} .
$$

Now

$g(l x)=\inf _{n \geq 1}\left\{\rho^{\frac{p_{n}}{K}}:\left(\sum_{m=1}^{\infty} \frac{1}{m^{r}}\left[M\left(\frac{\left|\phi_{m, n}(l x)\right|}{\rho}\right)\right]^{p_{m}}\right)^{\frac{1}{K}} \leq 1\right.$, uniformly in $\left.\mathrm{n}\right\} \rightarrow 0$, as $l \rightarrow 0$.

This completes the proof.

Theorem 2.3. Suppose that $0<p_{m} \leq t_{m}<\infty$ for each $m \in \mathbb{N}$ and $r \geq 0$. Then

(i) $B V_{\sigma}(M, p) \subseteq B V_{\sigma}(M, t)$,

(ii) $B V_{\sigma}(M) \subseteq B V_{\sigma}(M, r)$.

Proof. . [i] Suppose that $x \in B V_{\sigma}(M, p)$. This implies that

$\left[M\left(\frac{\left|\phi_{i, n}(x)\right|}{\rho}\right)\right]^{p_{m}} \leq 1$ for sufficiently large values of $i$, say $i \geq m_{0}$ for some fixed $m_{0} \in \mathbb{X}$. Since $M$ is non decreasing, we have

$$
\sum_{m=m_{0}}^{\infty}\left[M\left(\frac{\left|\phi_{i, n}(x)\right|}{\rho}\right)\right]^{t_{m}} \leq \sum_{m=m_{0}}^{\infty}\left[M\left(\frac{\left|\phi_{i, n}(x)\right|}{\rho}\right)\right]^{p_{m}}<\infty
$$

Hence $x \in B V_{\sigma}(M, t)$.

The proof of [ii] is trivial. 
The following result is a consequence of the above result.

Corollary 1. If $0<p_{m} \leq 1$ for each $m$, then $B V_{\sigma}(M, p) \subseteq B V_{\sigma}(M)$.

If $p_{m} \geq 1$ for all $m$, then $B V_{\sigma}(M) \subseteq B V_{\sigma}(M, p)$.

Theorem 2.4. . The sequence space $B V_{\sigma}(M, p, r)$ is solid.

Proof. Let $x \in B V_{\sigma}(M, p, r)$. This implies that

$$
\sum_{m=1}^{\infty} m^{-r}\left[M\left(\frac{\left|\phi_{k, n}(x)\right|}{\rho}\right)\right]^{p_{m}}<\infty .
$$

Let $\left(\alpha_{m}\right)$ be sequence of scalars such that $\left|\alpha_{m}\right| \leq 1$ for all $m \in \mathbb{X}$. Then the result follows from the following inequality

$$
\sum_{m=1}^{\infty} m^{-r}\left[M\left(\frac{\left|\alpha_{m} \phi_{k, n}(x)\right|}{\rho}\right)\right]^{p_{m}} \leq \sum_{m=1}^{\infty} m^{-r}\left[M\left(\frac{\left|\phi_{k, n}(x)\right|}{\rho}\right)\right]^{p_{m}}<\infty .
$$

Hence $\alpha x \in B V_{\sigma}(M, p, r)$ for all sequences of scalars $\left(\alpha_{m}\right)$ with $\left|\alpha_{m}\right| \leq 1$ for all $m \in$ $\mathbb{X}$ whenever $x \in B V_{\sigma}(M, p, r)$.

From Theorem 4 and Lemma we have :

Corollary 2. . The sequence space $B V_{\sigma}(M, p, r)$ is monotone.

Theorem 2.5. . Let $M_{1}, M_{2}$ be Orlicz functions satisfying $\triangle_{2}$ - condition and $r, r_{1}, r_{2} \geq 0$. Then we have

(i) If $r>1$ then $B V_{\sigma}\left(M_{1}, p, r\right) \subseteq B V_{\sigma}\left(M 0 M_{1}, p, r\right)$,

(ii) $B V_{\sigma}\left(M_{1}, p, r\right) \cap B V_{\sigma}\left(M_{2}, p, r\right) \subseteq B V_{\sigma}\left(M_{1}+M_{2}, p, r\right)$,

(iii) If $r_{1} \leq r_{2}$ then $B V_{\sigma}\left(M, p, r_{1}\right) \subseteq B V_{\sigma}\left(M, p, r_{2}\right)$.

Proof. [i] Since $M$ is continuous at 0 from right, for $\epsilon>0$ there exists $0<\delta<1$ such that $0 \leq c \leq \delta$ implies $M(c)<\epsilon$. If we define

$$
I_{1}=\left\{m \in \mathbb{N}: M_{1}\left(\frac{\left|\phi_{m, n}(x)\right|}{\rho}\right) \leq \delta \text { for some } \rho>0\right\},
$$




$$
I_{2}=\left\{m \in \mathbb{N}: M_{1}\left(\frac{\left|\phi_{m, n}(x)\right|}{\rho}\right)>\delta \text { for some } \rho>0\right\},
$$

then, when $M_{1}\left(\frac{\left|\phi_{m, n}(x)\right|}{\rho}\right)>\delta$ we get

$$
M\left(M_{1}\left(\frac{\left|\phi_{m, n}(x)\right|}{\rho}\right)\right) \leq\{2 M(1) / \delta\} M_{1}\left(\frac{\left|\phi_{m, n}(x)\right|}{\rho}\right) .
$$

Hence for $x \in B V_{\sigma}\left(M_{1}, p, r\right)$ and $r>1$

$$
\begin{aligned}
& \sum_{m=1}^{\infty} m^{-r}\left[M 0 M_{1}\left(\frac{\left|\phi_{m, n}(x)\right|}{\rho}\right)\right]^{p_{m}}=\sum_{m \in I_{1}} m^{-r}\left[M 0 M_{1}\left(\frac{\left|\phi_{m, n}(x)\right|}{\rho}\right)\right]^{p_{m}} \\
&+\sum_{m \in I_{2}} m^{-r}\left[M 0 M_{1}\left(\frac{\left|\phi_{m, n}(x)\right|}{\rho}\right)\right]^{p_{m}} \\
& \leq \sum_{m \in I_{1}} m^{-r}[\epsilon]^{p_{m}} \\
&+\sum_{m \in I_{2}} m^{-r}\left[\{2 M(1) / \delta\} M_{1}\left(\frac{\left|\phi_{m, n}(x)\right|}{\rho}\right)\right]^{p_{m}} \\
& \leq \max \left(\epsilon^{h}, \epsilon^{H}\right) \sum_{m=1}^{\infty} m^{-r} \\
&+\max \left(\{2 M(1) / \delta\}^{h},\{2 M(1) / \delta\}^{H}\right) \\
&\text { (where } \left.0<h=\inf p_{m} \leq p_{m} \leq H=\sup _{m} p_{m}<\infty\right) .
\end{aligned}
$$

[ii] The proof follows from the following inequality

$$
\begin{aligned}
m^{-r}\left[\left(M_{1}+M_{2}\right)\left(\frac{\left|\phi_{m, n}(x)\right|}{\rho}\right)\right]^{p_{m}} \leq & C m^{-r}\left[M_{1}\left(\frac{\left|\phi_{m, n}(x)\right|}{\rho}\right)\right]^{p_{m}} \\
& +C m^{-r}\left[M_{2}\left(\frac{\left|\phi_{m, n}(x)\right|}{\rho}\right)\right]^{p_{m}} .
\end{aligned}
$$

[iii ] The proof is straightforward.

Corollary 3. . Let $M$ be an Orlicz function satisfying $\triangle_{2}$ - condition. Then we have

(1) If $r>1$, then $B V_{\sigma}(p, r) \subseteq B V_{\sigma}(M, p, r)$,

(2) $B V_{\sigma}(M, p) \subseteq B V_{\sigma}(M, p, r)$,

(3) $B V_{\sigma}(p) \subseteq B V_{\sigma}(p, r)$,

(4) $B V_{\sigma}(M) \subseteq B V_{\sigma}(M, r)$,

The proof is straightforward. 
ÖZET: $B V_{\sigma}$ dizi uzayı, Mursaleem tarafından tanımlanmış ve incelenmiştir [9]. Bu çalışmada $B V_{\sigma}$ uzayını, $B V_{\sigma}(M, p, r)$ uzayına genişleterek bu uzaya ilişkin bazı özelikleri ve kapsama bağıntılarını elde ettik.

\section{References}

[1] Z.U. Ahmad and M. Mursaleen , An application of banach limits, Proc. Amer. Math.Soc. 103 (1983), 244 - 246.

[2] S.T. Chen, Geometry of Orlicz Spaces, Dissertationes Math. (The Institute of Mathematics, Polish Academy of Sciences) (1996).

[3] M. A. Krasnoselskii, and Rutickii, Ya. B, Convex Functions and Orlicz Spaces, (Gooningen: P. Nordhoff Ltd.) (1961) (translation).

[4] J. Lindenstrauss and L. Tzafriri, On Orlicz sequence spaces, Israel J. Math., 10 : 379-390 (1971).

[5] G.G. Lorentz, A contribution to the theory of divergent series, Acta Math. 80(1948) 167-190.

[6] W. A. Luxemburg, Banach Function Spaces, Thesis (Delft) (1955)

[7] L. Maligranda, Orlicz spaces and interpolation, Seminar in Math. 5, Campinas (1989)

[8] M.Mursaleen, Matrix transformations between some new sequence spaces, Houston J. Math., 9 (1983), 505- 509.

[9] M.Mursaleen ,On some new invariant matrix methods of summability, Quart. J. Math., Oxford (2) 34 (1983), 77-86 .

[10] J. Musielak , Orlicz Spaces and Modular spaces, Lecture Notes in Math. 1034 (Springer- Verlag) (1983).

[11] W. Orlicz, Ü ber Raume $\left(L^{M}\right)$, Bulletin International de l' Académie Polonaise des Sciences et des Letters, Série A, 93 - 107 (1936).

[12] R. A. Raimi, Invariant means and invariant matrix method of summability, Duke Math. J. ., 30 (1963), 81- 94.

[13] M. M . Rao and Z. D. Ren, Theory of Orlicz spaces (New York, Basel, Hong Kong: Marcel Dekker Inc.) (1991).

[14] P. Schafer, Infinite matrices and invariant means, Proc. Amer. Math.Soc. 36 (1972), $104-110$.

[15] A. Wilansky, Summability Through Functional Analysis, North-Holland Mathematical Studies 85, 1984.

Current address: Department of Mathematics, A. M. U. Aligarh-202002 INDIA,

E-mail address: vakhan@math.com, 